\title{
Investigation of the Dynamic Characteristics of the Manipulator of the Industrial Robot Technology
}

\author{
Victor Butenko ${ }^{1}$, Irina Davidova ${ }^{1}$, Philipp Pastukhov ${ }^{1}$ and Tatyana Bagdasaryan ${ }^{1}$ \\ ${ }^{1}$ Don State Technical University, Rostov-on-Don, 1 Gagarin Square, Russia 344000
}

\begin{abstract}
The article describes the results of the study of dynamic characteristics of the manipulator of technological industrial robot. In addition, formulas for determining the forces of the control action on the links of the manipulator when performing specific technological methods of finishing the surfaces of the part are obtained. The influence of control forces on the functioning and technical capabilities of the manipulator of technological industrial robot is revealed. Standard layout schemes of manipulators of technological industrial robots were developed, and the analysis of their dynamic characteristics with the determination of the control action forces is carried out. This made it possible to develop and create a specific design of the robot for finishing turning and diamond smoothing of shaft surfaces in the structure of the automatic line.
\end{abstract}

\section{Introduction}

Technological industrial robots (TIR) are being applied in modern metal working for performance of finishing operations [1-7], which can perform such types of work as diamond smoothing, running the surface of the part by roller or ball, finishing or fine turning of cylindrical surfaces, cleaning of surfaces from burrs, polishing, etc. The choice of the layout scheme of the manipulator, which is the basis of the TIR and performs a particular operation of the finishing of the part, is a rather complicated and complex task, taking into account both the force effect of the tool on the workpiece surface, and the permissible number of degrees of freedom, the mass of the links and their mutual position. Therefore, the identification of the determining dynamic characteristics of the TIR manipulator will allow in the future to choose the design schemes of the manipulators to perform specific technological methods of finishing the surfaces of the part or their complexes.

The manipulator, which implements the force effect of the tool on the workpiece surface, can be considered as a complex system with an additional connection. Using the Dalamber's principle, it is possible to construct a dynamic model of the manipulator containing the coupling reaction in an explicit form. The simplest scheme can be seen in fig.1. Dynamic studies provide an opportunity to identify some driving forces, which are developed by actuators of the manipulator and is conditioned by the processing of the parts. 


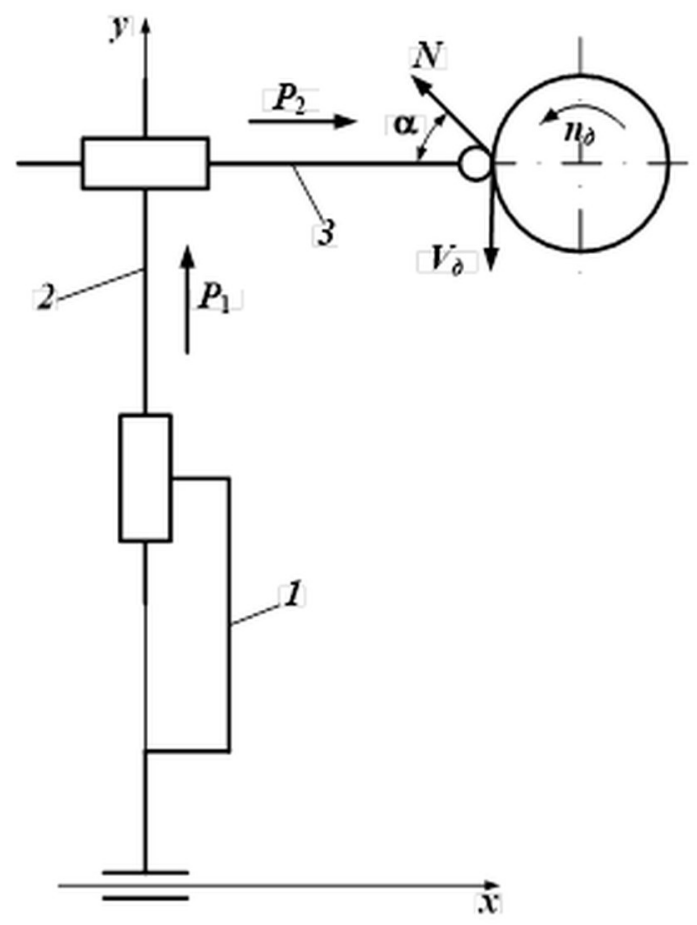

Fig. 1. The scheme of the manipulator, having a reaction connection of the tool with the processed surface of the part. 1 - stand, 2 - lifting mechanism, 3 - hand moving mechanism.

The task of the dynamic study of the manipulator was to determine the normal force $N$ at the point of contact of the tool with the workpiece surface and the rotation speed of the part $V_{\partial}$, which can be considered as the speed of movement of the tool on the treated surface. To solve this problem, the following values were taken as known: $m_{1}, m_{2}$ weight of the first and second links (modules) of the manipulator; $x, y$-coordinates of the centers of mass links in a fixed coordinate system $\mathrm{Oxy} ; G_{1}, G_{2}$ - gravity of the first and second links; P1, P2 - the forces developed by the drives of the manipulator to move its nodes and determine the process of processing the surface of the part.

According to the accepted scheme of the manipulator (Fig. 1) the angle of inclination of the normal force $\mathrm{N}$ to the fixed axis $\mathrm{x}$ of the coordinate system Oxy $\alpha$ is determined from the dependencies:

$$
\alpha=\operatorname{arctg}\left(N_{y} / N_{x}\right) \text { or } \alpha=\operatorname{arctg}\left(\left|V_{\partial x}\right| / V_{\partial y} \mid\right),
$$

where $N_{x}, N_{y}$ - the projection of the normal force $\mathrm{N}$ on the coordinate axis; $V_{\partial x}, V_{\partial y}-$ the projection of the velocity $V_{\partial}$ on the coordinate axis.

The equations of the manipulator motion can be represented as follows:

$$
\begin{aligned}
m_{2} \ddot{x} & =P_{2}-N \cos \alpha ; \\
\left(m_{1}+m_{2}\right) \ddot{y} & =P_{1}+N \sin \alpha+G_{1}+G_{2} .
\end{aligned}
$$

From equation (2) we can determine the normal force N:

$$
N=\left(P_{2}-m_{2} \ddot{x}\right) / \cos \alpha .
$$


If the expression (4) is differentiated by time, then the following differential equation is obtained, it provides the required type of transient by the force $\mathrm{N}$ :

$$
\dot{N}=\left[\left(P_{2}-m_{2} \dddot{x}\right) \cos \alpha+\dot{\alpha}\left(P_{2}-m_{2} \ddot{x}\right) \sin \alpha\right] / \cos ^{2} \alpha .
$$

If the normal force $N_{m p}$ at the point of contact of the tool with the workpiece surface is known, (depending on the processing method of the part, the value of this force can be calculated from the known components of the cutting force at the finish and fine turning $P_{x}$, $P_{y}, P_{z}$ or the force of indentation of the $P_{6}$ at diamond smoothing) the differential equation that provides the finishing process of the part can be expressed as follows:

$$
N=\lambda\left(N-N_{m p}\right),
$$

where $\lambda$ - the uncertain factor (Lagrange multiplier), which is found by solving the coupling equations for the control forces taking into account the stability conditions of the system "manipulator - tool - workpiece" [2].

Then the force of the control action $P_{2}$ is determined from the expression obtained by equating equations (5) and (6):

$$
\lambda\left(N-N_{m p}\right)=\left[\left(\dot{P}_{2}-m_{2} \dddot{x}\right) \cos \alpha+\dot{\alpha}\left(P_{2}-m_{2} \ddot{x}\right) \sin \alpha\right] / \cos ^{2} \alpha ;
$$

and then

$$
P_{2}=\frac{\lambda\left(N-N_{m p}\right) \cos ^{2} \alpha+m_{2}(\ddot{x} \cos \alpha+\ddot{x} \dot{\alpha} \sin \alpha)-\dot{P}_{2} \cos \alpha}{\dot{\alpha} \sin \alpha} .
$$

To determine the derivatives $\dddot{x}$ и $\dddot{y}$ one can use the following relationship between the velocity projections $V_{\partial}$ on the $x$ and $y$ coordinate axes:

$$
V_{\partial x}=V_{\partial y} \cdot \operatorname{tg} \alpha
$$

If in formula (9) put previously forestry dependence (1), (2), (4), (8) and doubledifferentiate the new expression for $V_{\partial x}$ in time, then the relationship between the derivatives $\dddot{x}$ and $\dddot{y}$ will have the following form:

$$
\dot{V}_{x}=\dddot{x}=\left[\cos ^{2} \alpha(\ddot{y} \dot{\alpha}+\ddot{\alpha} \dot{y})+2(\dot{\alpha})^{2} \dot{y} \cos \alpha \sin \alpha\right] \frac{1}{\cos ^{4} \alpha}+\frac{\ddot{y} \dot{\alpha}}{\cos ^{2} \alpha}+\dddot{y} \operatorname{tg} \alpha .
$$

The joint solution of the system consisting of equations (7) and (10) allows to obtain expressions for the determination of derivatives $\dddot{x}$ и $\dddot{y}$ :

$$
\begin{gathered}
\dddot{x}=\left[\dot{P}_{2}+\dot{\alpha}\left(P_{2}-m_{2} \ddot{x}\right) \operatorname{tg} \alpha-\lambda\left(N-N_{m p} \cos \alpha\right] \frac{1}{m_{2}} ;\right. \\
\dddot{y}=\left[\dot{P}_{2}-\lambda\left(N-N_{m p}\right) \cos \alpha\right] \frac{1}{m_{2} t g \alpha}+\left(P_{2}-m_{2} \ddot{x}\right) \frac{\dot{\alpha}}{m_{2}}-\frac{2 \ddot{y} \dot{\alpha}+\ddot{y}}{\sin \alpha \cos \alpha}-\frac{2(\dot{\alpha})^{2} \dot{y}}{\cos ^{2} \alpha} .
\end{gathered}
$$

The force of the control action $P_{1}$ is determined from the equation (3)

$$
P_{1}=\left(m_{1}+m_{2}\right) \ddot{y}-P_{2}-N \sin \alpha .
$$

If the expression (13) is differentiated by time and to transform, the following differential equation is obtained:

$$
\dot{N}=\left[\left(m_{1}+m_{2}\right) \dddot{y}-\dot{P}_{1}\right] \frac{1}{\sin \alpha}-\left[\left(m_{1}+m_{2}\right) \ddot{y}-P_{1}-G_{1}-G_{2}\right] \frac{\dot{\alpha} \cos \alpha}{\sin ^{2} \alpha} .
$$


Then, solving together the equations (3), (12) and (14), the formula for determining the force of the control action $P_{l}$, will have the following form:

$$
\begin{array}{r}
P_{1}=\frac{\lambda}{\dot{\alpha}}\left(N-N_{m p}\right)\left(\sin \alpha \operatorname{tg} \alpha+\cos \alpha \frac{m_{1}+m_{2}}{m_{2}}\right)-G_{1}-G_{2}+\left(m_{1}+m_{2}\right) \ddot{y}+\frac{\dot{P}_{1} \operatorname{tg} \alpha}{\dot{\alpha}}- \\
-\left(m_{1}+m_{2}\right) \operatorname{tg} \alpha\left[\frac{\dot{P}_{2}}{m_{2} \operatorname{tg} \alpha}+\left(P_{2}-m_{2} \ddot{x}\right) \frac{\dot{\alpha}}{m_{2}}-\frac{2 \ddot{y} \dot{\alpha}+\ddot{\alpha} \dot{y}}{\sin \alpha \cos \alpha}-\frac{2 \dot{y}(\dot{\alpha})^{2}}{\cos ^{2} \alpha}\right] \frac{1}{\omega} .
\end{array}
$$

Since the treatment of cylindrical surfaces of parts is considered, then $\dot{\alpha}=\omega=$ const and formula (15) is converted as follows:

$$
P_{1}=\frac{\lambda\left(N-N_{m p}\right)}{\omega}\left(\frac{\sin ^{2} \alpha}{\cos \alpha}+\frac{\left(m_{1}+m_{2}\right) \cos \alpha}{m_{2}}\right)-\frac{\left(m_{1}+m_{2}\right) \operatorname{tg} \alpha}{\omega}\left(\frac{\dot{P}_{2}}{m_{2} \operatorname{tg} \alpha}+\frac{P_{2} \omega}{m_{2}}-\frac{2 \omega^{2} \dot{y}}{\cos ^{2} \alpha}\right)+\frac{\dot{P}_{1} \operatorname{tg} \alpha}{\omega} .
$$

The expressions (14) and (16) for determining the control forces $P_{1}$ and $P_{2}$ are nonlinear vector functions of time, generalized coordinates expressed through derivatives $\dddot{x}$ and $\dddot{y}$, masses of links and velocities, which provide the output of the tool fixed in the grip of the manipulator to a given point in space with a given velocity vector $V_{\partial}$, which guarantees the desired property of the tool movement in speed and position.

Knowledge of the control forces $P_{1}$ and $P_{2}$ allows you to optimize the trajectory of movement of the links of the manipulator in degrees of freedom and can be used to determine the laws of their movement. It should be noted that the faster the movement of links, the sooner the manipulator will be released to perform new tasks, and the higher its performance will be. However, the rapid movement of the links requires a lot of effort, and efforts are limited by the strength of the parts of the manipulator and the power of its drive devices. Analysis of the formulas (14) and (16) leads to the conclusion that the choice of the layout scheme of the manipulator TIR depends not only on the set value of the normal force $N_{m p}$, due to the method and modes of processing parts, and the angular velocity of rotation of the workpiece $\omega$, but also on the ratio between the masses of the links $m_{1}$ and $m_{2}$, the number of degrees of freedom of the manipulator and the relative position of its links. Therefore, when choosing the layout scheme of the manipulator TIR to perform a specific technological operation, not only their technical capabilities, for example, by the force of pressing the $P_{6}$ or the component of the cutting force $P_{z}$ and the permissible speed of rotation of the workpiece $V_{\partial}$, but also the required forces of the control action $P_{1}, P_{2}$, which determine the laws of motion of the executive bodies of TIR and the required power of its drives.

In fig. 2 one can see the typical layout schemes of manipulators TIR horizontal movement of the hand with the tool, widely used in metalworking to perform operations diamond smoothing surfaces of parts, finishing and fine turning, deburring, scale and deposits with cylindrical and shaped surfaces of blanks and parts, as well as performing other technological operations. For them, the values of the control action forces $P_{1}$ and $P_{2}$ were analyzed, provided that for a particular method of finishing the part $\omega=$ const, and the corresponding formulas for their determination were obtained (table. 1). In the presented formulas, the parameter $\mathrm{q}$, depending on the trajectory of the tool, the processing modes and the ratio between force and mass, can be determined from the equations of motion of the manipulator given in [8-12], by converting them in accordance with the accepted layout scheme. 


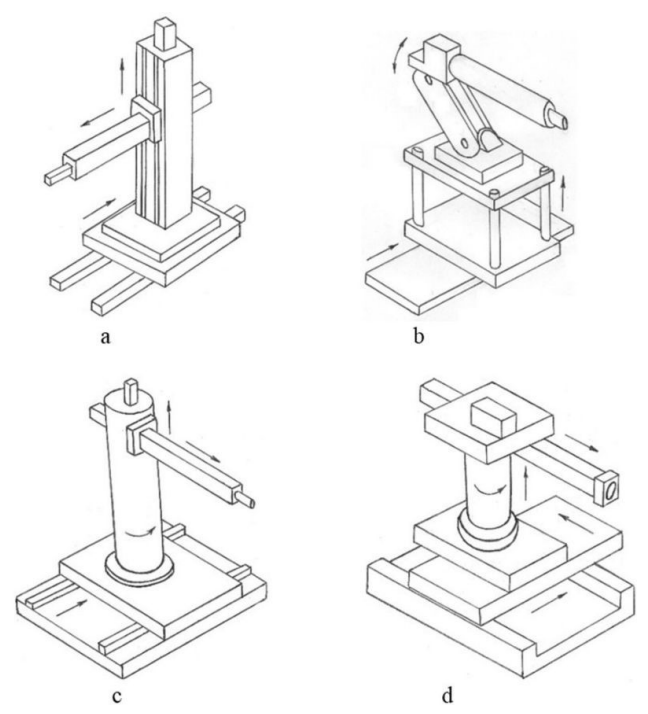

Fig. 2. Typical layout diagrams of manipulators TIR horizontal displacement with 3 (a, b), 4 (c) and 5 (d) degrees of freedom.

Table 1. The values of the control action forces $P_{1}$ and $P_{2}$ for different layout schemes, TIR

\begin{tabular}{|c|c|c|c|c|c|c|}
\hline \multirow[t]{2}{*}{$\begin{array}{l}\text { Scheme } \\
\text { of TIR }\end{array}$} & \multicolumn{2}{|c|}{$\begin{array}{l}\text { diamond } \\
\text { smoothing }\end{array}$} & \multicolumn{2}{|c|}{ final turning } & \multicolumn{2}{|c|}{ Driving forces } \\
\hline & $\begin{array}{c}P_{6}, \\
\mathrm{H}\end{array}$ & $\begin{array}{l}V_{\partial} \\
\mathrm{M} / \mathrm{c}\end{array}$ & $P_{z}, \mathrm{H}$ & $V_{\partial}, \mathrm{M} / \mathrm{c}$ & $P_{1}$ & $P_{2}$ \\
\hline \multirow[t]{2}{*}{$a$} & $\begin{array}{l}200- \\
1500\end{array}$ & $\begin{array}{c}0,1- \\
0,5\end{array}$ & & & $0,36 \frac{\left(0,4 m_{1}+0,5 m_{2}\right) q}{\omega}$ & $1,02 \frac{0,5 m_{2} q}{\omega}+P_{6}$ \\
\hline & & & $\begin{array}{l}500- \\
2500\end{array}$ & $0,1-0,5$ & $0,41 \frac{\left(0,4 m_{1}+0,5 m_{2}\right) q}{\omega}$ & $1,18 \frac{0,5 m_{2} q}{\omega}+P_{z}$ \\
\hline \multirow[t]{2}{*}{$b$} & $\begin{array}{l}100- \\
800\end{array}$ & $\begin{array}{c}0,01- \\
0,3\end{array}$ & & & $0,24 \frac{\left(0,5 m_{1}+m_{2}\right) q}{\omega}$ & $1,15 \frac{m_{2} q}{\omega}+P_{в}$ \\
\hline & & & $\begin{array}{l}300- \\
1200\end{array}$ & $\begin{array}{c}0,05- \\
0,3\end{array}$ & $0,39 \frac{\left(0,5 m_{1}+m_{2}\right) q}{\omega}$ & $0,97 \frac{m_{2} q}{\omega}+P_{z}$ \\
\hline \multirow[t]{2}{*}{$c$} & $\begin{array}{l}200- \\
1500\end{array}$ & $\begin{array}{c}0,1- \\
0,5\end{array}$ & & & $0,28 \frac{\left(m_{1}+0,6 m_{2}\right) q}{\omega}$ & $1,45 \frac{0,8 m_{2} q}{\omega}+P_{в}$ \\
\hline & & & $\begin{array}{l}400- \\
2000\end{array}$ & $0,1-0,5$ & $0,32 \frac{\left(m_{1}+0,6 m_{2}\right) q}{\omega}$ & $1.56 \frac{0,8 m_{2} q}{\omega}+P_{z}$ \\
\hline \multirow[t]{2}{*}{$d$} & $\begin{array}{l}100- \\
1600\end{array}$ & $\begin{array}{c}0,1- \\
0,8\end{array}$ & & & $0,47 \frac{\left(0,5 m_{1}+0,6 m_{2}\right) q}{\omega}$ & $0,94 \frac{0,5 m_{2} q}{\omega}+P_{z}$ \\
\hline & & & $\begin{array}{l}300- \\
2500\end{array}$ & $0,1-0,8$ & $0,55 \frac{\left(0,5 m_{1}+0,6 m_{2}\right) q}{\omega}$ & $1,08 \frac{0,5 m_{2} q}{\omega}+P_{z}$ \\
\hline
\end{tabular}




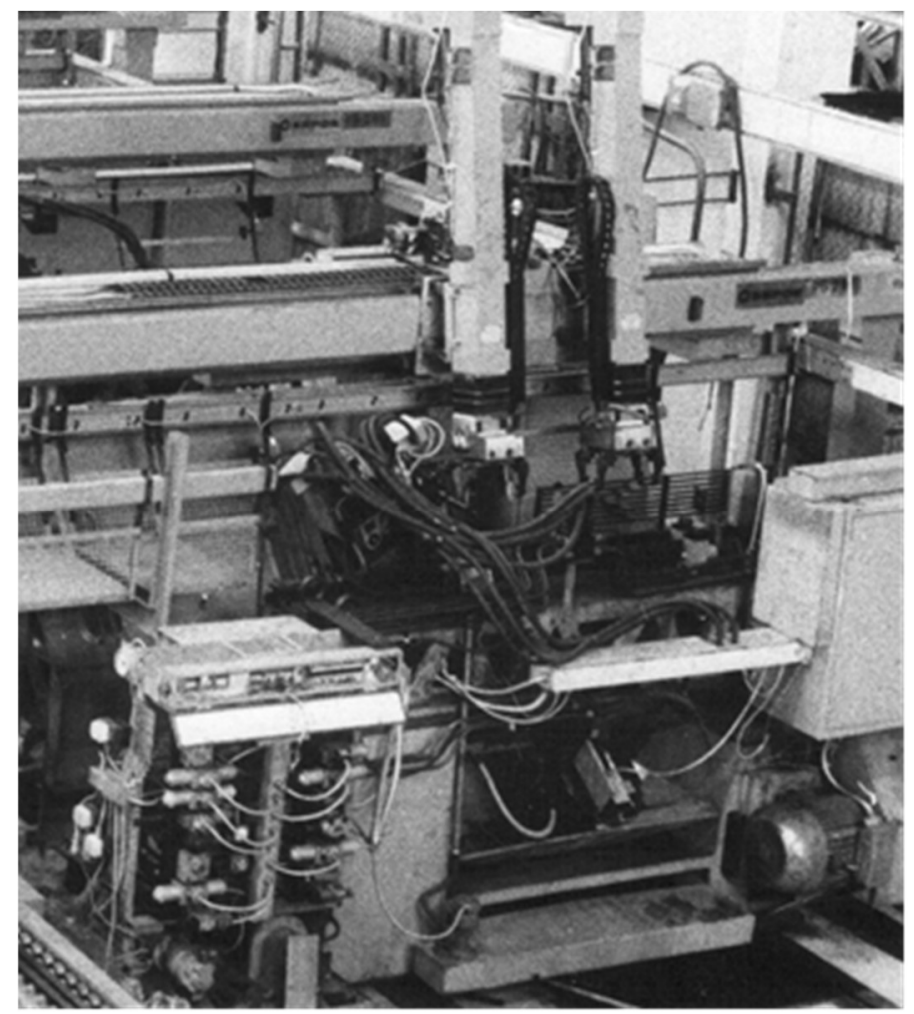

Fig 3. General view of the TIR for finishing surfaces of parts, built-in automatic line.

The analysis of the values of forces $P_{1}, P_{2}$ and technical characteristics of the manipulators allowed us to develop and create a specific design for finishing TIR (fine) turning, diamond smoothing of shaft surfaces and other technological operations of finishing parts in the structure of the automatic line (Fig. 3). The basis was the layout scheme of the manipulator which is presented in Fig. 2,a.

The production tests of the developed design of the TIR showed that their use in diamond smoothing of the surfaces of parts and performing cleaning operations can reduce the spread of the roughness parameter $R a$ and micro-hardness of the surface layer material by $20-30 \%$ and more than 1.5 times increase the productivity of operations related to the removal of burrs and scale from surfaces and forgings of iron-carbon alloys [13-15].

\section{References}

1. S.A. Kryukov, M.A. Tkach: Procedia Engineering, 206, 200 (2017)

2. J. Zeisig, N. Schädlich, L. et.al, RG Journal Impact Rankings, 382:383, 107 (2017)

3. L.V. Shipulin, A.A. Dyakonov, M.M. Al-Aqeeli: Procedia Engineering, 206, 1211 (2017)

4. H. Yamaguchi, J. Kang, F. Hashimoto: CIRP Annals, 60:1, 339 (2011)

5. Ioan D. Marinescu, W. Brian Rowe, Boris Dimitrov, Ichiro Inasaki: Tribology of Abrasive Machining Processes, 369 (2004)

6. V.M. Shumyacher, P.U. Bochkarev, A.V. Slavin: Procedia Engineering, 206, 232 (2017) 
7. Eugene Averin. Mining. Engineering, 3:6, 888 (December 2017)

8. E.S. Kiselev: Study guide, 186 (2003)

9. V.G. Poltoratskiy, V.I. Lavrinenko, M.N. Safonova, G.A. Petasyuk: Diamond and Related Materials, 68, 66 (September 2016)

10. Valentina K. Prokudina: Concise Encyclopedia of Self-Propagating High-Temperature Synthesis, 2 (2017)

11. V.N. Latyshev, A.G. Naumov, V.S., Radnyuk, et al.: Metalworking, 3:45, 9 (2008)

12. V.I. Butenko: Rostov-on-Don: DSTU Publ. Center, 219 (2016)

13. Liu Yang, Deguo Wang, Yanbao Guo: Tribology International, 123, 180 (2018)

14. Bhavesh Gupta, Amit Jain, Rajesh Purohit, R.S. Rana, Bhupendra Gupta: Materials Today: Proceedings, 5:9-3, 17725 (2018)

15. V.I. Butenko: Rostov-on-Don: DSTU, 160 (2018) 